\title{
Analysis of urinary oligosaccharides in lysosomal storage disorders by capillary high-performance anion-exchange chromatography-mass spectrometry
}

\author{
Cees Bruggink • Ben J. H. M. Poorthuis • \\ André M. Deelder • Manfred Wuhrer
}

Received: 13 January 2012 /Revised: 14 March 2012 / Accepted: 22 March 2012 /Published online: 20 April 2012

(C) The Author(s) 2012. This article is published with open access at Springerlink.com

\begin{abstract}
Many lysosomal storage diseases are characterized by an increased urinary excretion of glycoconjugates and oligosaccharides that are characteristic for the underlying enzymatic defect. Here, we have used capillary highperformance anion-exchange chromatography (HPAEC) hyphenated to mass spectrometry to analyze free oligosaccharides from urine samples of patients suffering from the lysosomal storage disorders fucosidosis, $\alpha$-mannosidosis, $\mathrm{G}_{\mathrm{M} 1}$-gangliosidosis, $\mathrm{G}_{\mathrm{M} 2}$-gangliosidosis, and sialidosis. Glycan fingerprints were registered, and the patterns of accumulated oligosaccharides were found to reflect the specific blockages of the catabolic pathway. Our analytical approach allowed structural analysis of the excreted oligosaccharides and revealed several previously unpublished oligosaccharides. In conclusion, using online coupling of HPAEC with mass spectrometric detection, our study provides characteristic urinary oligosaccharide fingerprints with diagnostic potential for lysosomal storage disorders.
\end{abstract}

Electronic supplementary material The online version of this article (doi:10.1007/s00216-012-5968-9) contains supplementary material, which is available to authorized users.

C. Bruggink $\cdot$ A. M. Deelder $\cdot$ M. Wuhrer

Biomolecular Mass Spectrometry Unit,

Department of Parasitology, Leiden University Medical Center,

P.O. Box 9600, 2300 RC Leiden, The Netherlands

B. J. H. M. Poorthuis

Department of Medical Biochemistry, Academic Medical Center,

Meibergdreef 15,

1105 AZ Amsterdam, The Netherlands

C. Bruggink $(\square)$

Thermo Fisher Scientific,

Abberdaan 114,

1046 AA Amsterdam, The Netherlands

e-mail: c.bruggink@lumc.nl
Keywords HPAEC-IPAD · Catabolism · Metabolic disorder $\cdot$ Clinical glycomics $\cdot N$-linked glycans $\cdot$ Glycolipids

\section{Introduction}

Fucosidosis, $\alpha$-mannosidosis, $\mathrm{G}_{\mathrm{M} 1}$-gangliosidosis, $\mathrm{G}_{\mathrm{M} 2}-$ gangliosidosis, and sialidosis are autosomal recessive lysosomal storage diseases (LSD). These LSDs are the result of defects of one or more enzymes or cofactors involved in the catabolism of glycoconjugates that takes place in the lysosome. Fucosidosis is caused by a deficient lysosomal $\alpha$-Lfucosidase (EC 3.2.1.51) and results in secretion of fucosyloligosaccharides $[1,2]$. Deficient lysosomal $\alpha$-D-mannosidase (EC 3.2.1.24) causes $\alpha$-mannosidosis and excessive urinary excretion of oligomannosidic glycans [3-5]. Sialidosis is caused by deficient acid exo- $\alpha$-sialidase (EC 3.2.1.18) [6]. The urinary excretion of sialyloligosaccharides is similar to that found in galactosialidosis $[1,7] . \mathrm{G}_{\mathrm{M} 1}$-gangliosidosis is a neurosomatic disease due to the deficient activity of $\beta$ galactosidase (EC 3.2.1.23) [8, 9]. In addition to the storage of $\mathrm{G}_{\mathrm{M} 1}$-gangliosides, glycoconjugates with $\beta$-galactose at the non-reducing end are increased in patients' urine.

$\mathrm{G}_{\mathrm{M} 2}$-gangliosidosis is a group of three disorders (1) TaySachs disease, (2) Sandhoff disease, and (3) AB variant. For all variants of $\mathrm{G}_{\mathrm{M} 2}$-gangliosidosis, the major neural storage compound is ganglioside $\mathrm{G}_{\mathrm{M} 2}$ [10-12]. Only in Sandhoff disease oligosaccharides derived from glycoproteins accumulate due to the deficiency of $\beta$-hexosaminidase $\mathrm{A}$ in addition to the (functional) deficiency of $\beta$-hexosaminidase $B$ [13]. Blockage of the $\mathrm{N}$-glycan catabolism results in accumulation of oligosaccharides carrying a single $\mathrm{N}$-acetylglucosamine residue at the nonreducing end in tissues and urine of Sandhoff disease patients [14-16]. The current study includes the analysis of urine samples of patients suffering from Sandhoff disease. 
Biochemical screening of these LSDs is usually performed using thin-layer chromatography (TLC) [17-19], since TLC is relatively easy to perform and does not require expensive equipment. However, interpretation of a TLC pattern of excreted oligosaccharides requires much experience in pattern recognition. On the other hand, liquid chromatography combined with UV [20] or fluorescence [21] detection is easier to reproduce and to interpret $[22,23]$.

Hyphenation of liquid chromatography with mass spectrometry allows the detailed characterization of oligosaccharides [24]. We have previously described a capillary high-performance anion-exchange chromatograph (HPAEC) setup with combined integrated pulsed amperometric (IPAD) and ion trap mass spectrometric detection which was used to characterize oligosaccharides from urine of $\mathrm{G}_{\mathrm{M} 1}$-gangliosidosis [25] and galactosialidosis [26] patients. This combination of chromatography IPAD and mass spectrometric detection allows detailed glycan analysis and characterization, when compared with TLC, HPLC, or HPAECIPAD without mass spectrometry (MS). Using this analytical setup, we report on the analysis of oligosaccharides in urine samples of fucosidosis, $\alpha$-mannosidosis, $\mathrm{G}_{\mathrm{M} 1}$-gangliosidosis, $\mathrm{G}_{\mathrm{M} 2}$-gangliosidosis, and sialidosis patients. The results provided in glycan fingerprints that are found to be characteristic for the individual diseases and reflect the specific enzymatic defects.

\section{Materials and methods}

\section{Materials}

Analytical-reagent-grade sodium hydroxide $(50 \% w / w)$, sodium acetate, sulfuric acid, and sodium chloride were obtained from J.T. Baker (Deventer, The Netherlands). Acetonitrile was from Biosolve (Valkenswaard, The Netherlands). All solutions were prepared using water from a Milli-Q synthesis system from Millipore BV (Amsterdam, The Netherlands). Details on urine samples are given in Table 1.

\section{Sample preparation}

Oligosaccharides of the samples were isolated with graphitized carbon solid-phase extraction, as described previously [27]. A $200-\mu \mathrm{L}$ sample was diluted with $1,800 \mu \mathrm{L}$ water and loaded on a Carbograph SPE (210142) from Alltech Associates Inc. (Deerfield, IL). The cartridge was washed with $6 \mathrm{~mL}$ of demineralized water. The oligosaccharides were eluted from the column with $3 \mathrm{~mL}$ of $25 \%$ acetonitrile containing $0.05 \%$ trifluoroacetic acid. The eluate was evaporated under a nitrogen stream at room temperature until the volume was decreased by $50 \%$. The remaining solution was lyophilized and reconstituted with $200 \mu \mathrm{L}$ water.
Table 1 Information about the urine samples

\begin{tabular}{lllll}
\hline Sample code & Disorder & Sex & $\begin{array}{l}\text { Age } \\
\text { (years) }\end{array}$ & $\begin{array}{l}\text { Creatinine } \\
(\mathrm{mmol} / \mathrm{L})\end{array}$ \\
\hline $\mathrm{U} 1$ & Fucosidosis & $\mathrm{M}$ & 18 & 2.22 \\
$\mathrm{U} 2$ & $\mathrm{G}_{\mathrm{M} 1}$-gangliosidosis & $\mathrm{F}$ & 0.42 & 1.04 \\
$\mathrm{U} 3$ & $\mathrm{G}_{\mathrm{M} 2}$-gangliosidosis & $\mathrm{M}$ & 0.75 & 5.37 \\
$\mathrm{U} 4$ & $\mathrm{G}_{\mathrm{M} 2}$-gangliosidosis & $\mathrm{M}$ & 0.58 & 1.04 \\
$\mathrm{U} 5$ & $\alpha$-Mannosidosis & $\mathrm{M}$ & 22 & 18.86 \\
$\mathrm{U} 6$ & $\alpha$-Mannosidosis & $\mathrm{F}$ & 7 & 8.46 \\
$\mathrm{U} 7$ & $\alpha$-Mannosidosis & $\mathrm{M}$ & 20 & 14.52 \\
$\mathrm{U} 8$ & Sialidosis & $\mathrm{F}$ & Unknown & Not determined
\end{tabular}

The age at the time point of sample gathering is given

\section{Capillary HPAEC}

The capillary chromatographic system consists of a modified Dionex BioLC system from Thermo Fisher Scientific (Sunnyvale, CA, USA) comprising a microbore GP40 gradient pump, a Famos micro-autosampler with a full PEEK (polyether ether ketone) injector equipped with a $1 \mu \mathrm{L}$ loop and an ED40 electrochemical detector. BioLC control, data acquisition from the ED40 detector, and signal integration was supported by Dionex Chromeleon software (Themo Fisher Scientific). This modified system has been described in detail before [25]. A prototype capillary column (250× $0.4 \mathrm{~mm}$ I.D.) packed with CarboPac PA200 resin was manufactured by Thermo Fisher Scientific. The GP40 eluent flow was split by a homemade PEEK splitter to $10 \mu \mathrm{L} \mathrm{min}$. The pump was provided with the following eluents: eluent A, water; eluent B, $500 \mathrm{mM}$ sodium hydroxide; eluent C, $500 \mathrm{mM}$ sodium acetate. All separations were performed at room temperature. The following ternary gradient was used for separating oligosaccharides of fucosidosis, $\mathrm{G}_{\mathrm{M} 2}$-gangliosidosis, and sialidosis- $76 \% \mathrm{~A}+24 \% \mathrm{~B}$ ( -20 to $-14 \mathrm{~min}$ ) isocratic sodium hydroxide column wash; $88 \% \mathrm{~A}+12 \% \mathrm{~B}$ (-14 to $0 \mathrm{~min}$ ) isocratic equilibration of the column; a linear sodium acetate gradient $(0-55 \mathrm{~min})$ to $25.5 \% \mathrm{~A}+12 \% \mathrm{~B}+62.5 \% \mathrm{C}$ was used for the separation. For separating oligosaccharides of $\alpha$-mannosidosis and $\mathrm{G}_{\mathrm{M} 1 \text {-gangliosidosis, the following ternary gradient was }}$ used- $-76 \% \mathrm{~A}+24 \% \mathrm{~B}(-20$ to $-14 \mathrm{~min})$ isocratic sodium hydroxide column wash; $88 \% \mathrm{~A}+12 \% \mathrm{~B}(-14$ to $0 \mathrm{~min}$ ) isocratic equilibration of the column; linear sodium hydroxide gradient (0 to $9.1 \mathrm{~min}$ ) to $60 \% \mathrm{~A}+40 \% \mathrm{~B} ; 60 \% \mathrm{~A}+40 \%$ $\mathrm{B}$ (9.1 to $12.5 \mathrm{~min})$ isocratic; linear gradient (12.5 to $21.6 \mathrm{~min})$ to $85.2 \% \mathrm{~A}+12 \% \mathrm{~B}+2.8 \% \mathrm{C}$; linear sodium acetate gradient (21.6 to $104 \mathrm{~min}$ ) to $60.5 \% \mathrm{~A}+12 \% \mathrm{~B}+$ $27.5 \%$ C. Samples were injected at time $0.0 \mathrm{~min}$.

The ED40 detector applies the following waveform to the electrochemical cell $-E_{1}=0.1 \mathrm{~V}\left(t_{\mathrm{d}}=0.00-0.20 \mathrm{~s}, t_{1}=0.20\right.$ $0.40 \mathrm{~s}), E_{2}=-2.0 \mathrm{~V}\left(t_{2}=0.41-0.42 \mathrm{~s}\right), E_{3}=0.6 \mathrm{~V}\left(t_{3}=0.43 \mathrm{~s}\right)$, 
$E_{4}=-0.1 \mathrm{~V}\left(t_{4}=0.44-0.50 \mathrm{~s}\right)$ versus an $\mathrm{Ag} / \mathrm{AgCl}$ reference electrode [28]. A $1 \mathrm{~mm}$ gold work electrode and a $25 \mu \mathrm{m}$ gasket were installed.

\section{Mass spectrometry}

Coupled to the chromatographic system was an Esquire 3000 ion trap mass spectrometer from Bruker Daltonics (Bremen, Germany), equipped with an electrospray ionization source. To convert the HPAEC eluate into an ESI compatible solution, an in-line prototype desalter (Thermo Fisher Scientific) was used which was continuously regenerated with $12.5 \mathrm{mM}$ sulfuric acid at a flow rate of $0.8 \mathrm{~mL} \mathrm{~min}^{-1}$ [25]. A modified microbore AGP-1 (Thermo Fisher Scientific) was used as an auxiliary pump: To obtain efficient ionization of the eluted carbohydrates in the positive mode, $0.6 \mathrm{mM} \mathrm{NaCl}$ in $50 \%$ acetonitrile was pumped into the eluent flow via a MicroTEE (P-775 Upchurch Scientific, Oak Harbor, WA, USA) at a flow rate of $4.6 \mu \mathrm{L} \mathrm{min}^{-1}$. The mixture was directed to the electrospray ionization interface of the Esquire 3000 used in the positive mode. The MS was operated at the following conditions: dry temperature $325^{\circ} \mathrm{C}$, nebulizer $103 \mathrm{kPa}$, dry gas $71 \mathrm{~min}^{-1}$, capillary voltage $-3,500 \mathrm{~V}$, target mass $m / z 850$, scan speed $13,000 \mathrm{~m} / \mathrm{z} / \mathrm{s}$ in MS mode, scan range $150-2,000 \mathrm{~m} / z$, ICC target 50,000 with maximum accumulation time $50 \mathrm{~ms}$. For tandem MS, automatic selection of three precursors was applied with absolute intensity threshold 10,000 and $5 \%$ relative intensity threshold (relative to the base peak intensity), using fragmentation settings of $1.40 \mathrm{~V}$ with smart fragmentation amplification of $30 \%-100 \%$ and a fragmentation time of $40 \mathrm{~ms}$.

System suitability check

To check the correct functioning of the complete instrumental setup, every sequence started with analyzing a $50 \mathrm{nmol} \mathrm{mL}{ }^{-1}$ lactose solution with $60 \mathrm{mM} \mathrm{NaOH}$ as eluent. The resulting MS chromatogram should pass the following criteria: The retention time has to be $7.5 \mathrm{~min} \pm 15 \%$; in the total ion current chromatogram, the baseline level intensity should be $\leq 4.5$ e 6 with the noise intensity $\leq 7.5 \mathrm{e} 5$; for the extracted ion chromatogram $(\mathrm{m} / \mathrm{z} 365 \pm 0.5)$, the peak height intensity $\geq 4.5$ e6 with a peak width at half height of $\leq 55 \mathrm{~s}$.

\section{Data analysis}

MS as well as MS/MS spectra were manually interpreted using DataAnalysis (version 3.3, Bruker Daltonics). The extracted ion chromatograms (EIC) were used in order to determine the peak area of oligosaccharides present in the MS spectra. Signals of all detected charge states and isomers corresponding to the same compound were added up. Peak areas were normalized to the sum of all glycan peak areas of one sample.

\section{Results}

Free oligosaccharides from eight urine samples of patients suffering from various LSDs including fucosidosis, $\alpha$-mannosidosis, $\mathrm{G}_{\mathrm{M} 1}$-gangliosidosis, $\mathrm{G}_{\mathrm{M} 2}$-gangliosidosis, and sialidosis (Table 1) were analyzed by HPAECIPAD-MS to investigate disease-related, excreted degradation products. A total of 54 glycans were analyzed in these urine samples as sodium adducts using positive ion mode mass spectrometry. The set of 54 glycans was established by manual assignment of glycan species from all HPAEC-MS(/MS) data. This set includes glycans described previously in literature for the LSDs included in this study [2, 9, 15, 16, 25, 29-40] as well as the glycans found previously for galactosialidosis samples [26].

Urinary glycans in fucosidosis

Eight fucosylated oligosaccharides were detected in the urine sample of a fucosidosis patient, and the EIC of four of these fucosyl oligosaccharides are shown in Fig. 1. The neutral, fucosylated oligosaccharides were observed in an early retention time window (7 to $15 \mathrm{~min}$ ), while the acidic species HNSF resulted in signals between 22 and $25 \mathrm{~min}$. In order to enable relative quantification of the oligosaccharides, the signals were normalized to the overall intensity of

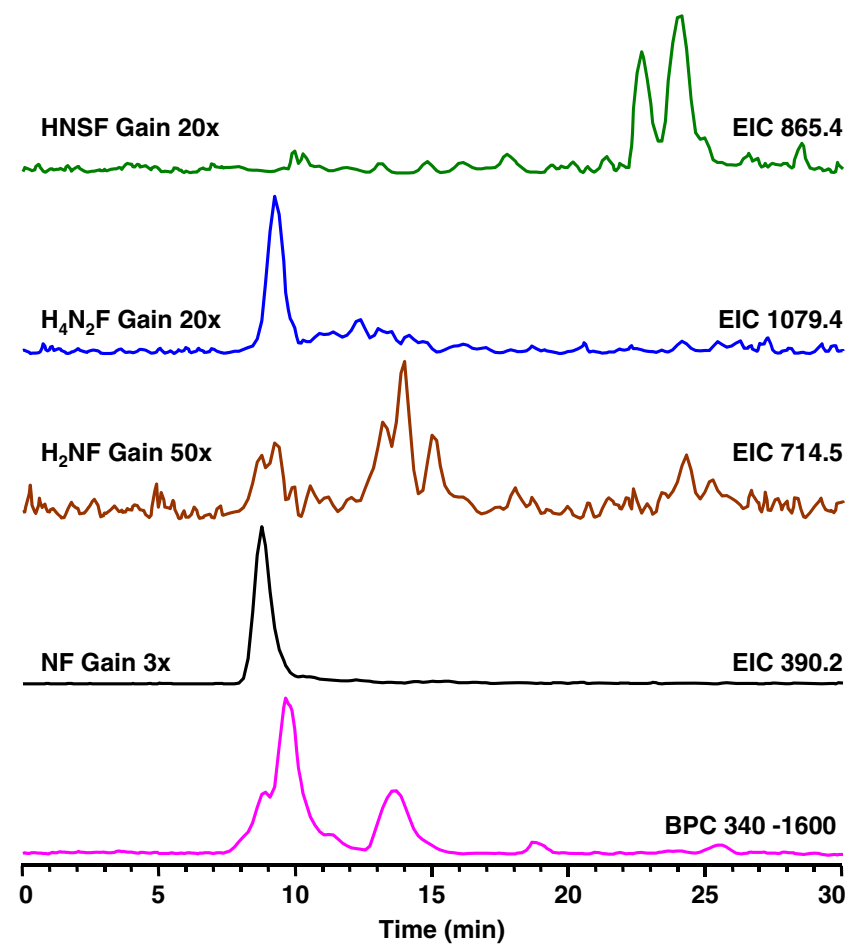

Fig. 1 Separation of oligosaccharides in urine of a fucosidosis patient. $H$ hexose, $N \mathrm{~N}$-acetylhexosamine, $F$ fucose, $S \mathrm{~N}$-acetylneuraminic acid, $B P C$ base peak chromatogram 
Table 2 Oligosaccharide species detected in urine samples U1-U8 and their relative area

\begin{tabular}{|c|c|c|c|c|c|c|c|c|c|c|}
\hline \multirow[t]{3}{*}{ Comp. } & \multirow{3}{*}{$\begin{array}{l}\text { Registered } \\
\mathrm{m} / \mathrm{z}\end{array}$} & \multirow[t]{3}{*}{ Charge state } & \multicolumn{8}{|c|}{ Relative area } \\
\hline & & & \multirow{2}{*}{$\begin{array}{l}\text { Fuc. } \\
\text { U1 }\end{array}$} & \multirow{2}{*}{$\begin{array}{l}\mathrm{G}_{\mathrm{M} 1} \text {-gang. } \\
\mathrm{U} 2\end{array}$} & \multicolumn{2}{|c|}{$\mathrm{G}_{\mathrm{M} 2}$-gangliosidosis } & \multicolumn{3}{|c|}{$\alpha$-Mannosidosis } & \multirow{2}{*}{$\begin{array}{l}\text { Sial. } \\
\text { U8 }\end{array}$} \\
\hline & & & & & U3 & U4 & U5 & U6 & U7 & \\
\hline $\mathrm{HF}$ & 349.2 & {$[\mathrm{M}+\mathrm{Na}]^{+}$} & $2.6 \%$ & $0.6 \%$ & $0.7 \%$ & $16.0 \%$ & $1.9 \%$ & $1.6 \%$ & $2.1 \%$ & $1.7 \%$ \\
\hline $\mathrm{H}_{2} \mathrm{~F}$ & 511.3 & {$[\mathrm{M}+\mathrm{Na}]^{+}$} & $0.3 \%$ & $4.4 \%$ & $0.5 \%$ & $0.7 \%$ & $0.2 \%$ & $0.0 \%$ & $0.3 \%$ & $2.8 \%$ \\
\hline $\mathrm{NF}$ & 390.2 & {$[\mathrm{M}+\mathrm{Na}]^{+}$} & $10.6 \%$ & $0.0 \%$ & $0.2 \%$ & $0.0 \%$ & $0.1 \%$ & $0.0 \%$ & $0.0 \%$ & $0.3 \%$ \\
\hline $\mathrm{HNF}$ & 552.5 & {$[\mathrm{M}+\mathrm{Na}]^{+}$} & $14.1 \%$ & $0.6 \%$ & $0.5 \%$ & $0.2 \%$ & $0.6 \%$ & $0.2 \%$ & $1.2 \%$ & $1.2 \%$ \\
\hline $\mathrm{H}_{2} \mathrm{NF}$ & 714.5 & {$[\mathrm{M}+\mathrm{Na}]^{+}$} & $0.3 \%$ & $0.9 \%$ & $0.6 \%$ & $0.8 \%$ & $0.9 \%$ & $0.3 \%$ & $1.0 \%$ & $1.1 \%$ \\
\hline $\mathrm{H}_{2} \mathrm{NF}_{2}$ & 860.5 & {$[\mathrm{M}+\mathrm{Na}]^{+}$} & $2.3 \%$ & $0.0 \%$ & $0.2 \%$ & $0.1 \%$ & $0.7 \%$ & $3.2 \%$ & $0.7 \%$ & $0.0 \%$ \\
\hline $\mathrm{H}_{3} \mathrm{~N}_{2} \mathrm{~F}$ & 1079.4 & {$[\mathrm{M}+\mathrm{Na}]^{+}$} & $1.3 \%$ & $0.0 \%$ & $0.0 \%$ & $0.0 \%$ & $0.0 \%$ & $0.0 \%$ & $0.0 \%$ & $0.0 \%$ \\
\hline HNSF & 865.4 & {$[\mathrm{M}-\mathrm{H}+2 \mathrm{Na}]^{+}$} & $2.3 \%$ & $0.0 \%$ & $0.3 \%$ & $0.0 \%$ & $0.0 \%$ & $0.0 \%$ & $0.0 \%$ & $0.0 \%$ \\
\hline $\mathrm{H}_{2} \mathrm{~N}_{2}$ & 771.5 & {$[\mathrm{M}+\mathrm{Na}]^{+}$} & $1.6 \%$ & $0.0 \%$ & $7.6 \%$ & $7.6 \%$ & $0.2 \%$ & $0.1 \%$ & $0.1 \%$ & $0.3 \%$ \\
\hline $\mathrm{H}_{3} \mathrm{~N}_{2}$ & $933.5 ; 478.3$ & {$[\mathrm{M}+\mathrm{Na}]^{+} ;[\mathrm{M}+2 \mathrm{Na}]^{2+}$} & $0.0 \%$ & $16.2 \%$ & $0.6 \%$ & $0.4 \%$ & $0.1 \%$ & $0.2 \%$ & $0.1 \%$ & $6.5 \%$ \\
\hline $\mathrm{H}_{4} \mathrm{~N}_{2}$ & 1095.5 & {$[\mathrm{M}+\mathrm{Na}]^{+}$} & $0.7 \%$ & $0.7 \%$ & $0.0 \%$ & $0.0 \%$ & $0.1 \%$ & $0.0 \%$ & $0.0 \%$ & $0.0 \%$ \\
\hline $\mathrm{H}_{4} \mathrm{~N}_{3}$ & $1298.5 ; 660.9$ & {$[\mathrm{M}+\mathrm{Na}]^{+} ;[\mathrm{M}+2 \mathrm{Na}]^{2+}$} & $0.0 \%$ & $0.5 \%$ & $0.0 \%$ & $0.0 \%$ & $0.0 \%$ & $0.0 \%$ & $0.0 \%$ & $0.0 \%$ \\
\hline $\mathrm{H}_{3} \mathrm{~N}_{3}$ & $1136.5 ; 580.0$ & {$[\mathrm{M}+\mathrm{Na}]^{+} ;[\mathrm{M}+2 \mathrm{Na}]^{2+}$} & $0.0 \%$ & $0.0 \%$ & $8.0 \%$ & $8.2 \%$ & $0.0 \%$ & $0.0 \%$ & $0.0 \%$ & $0.0 \%$ \\
\hline $\mathrm{H}_{3} \mathrm{~N}_{4}$ & $1339.4 ; 681.2$ & {$[\mathrm{M}+\mathrm{Na}]^{+} ;[\mathrm{M}+2 \mathrm{Na}]^{2+}$} & $0.0 \%$ & $0.0 \%$ & $14.8 \%$ & $15.5 \%$ & $0.0 \%$ & $0.0 \%$ & $0.0 \%$ & $0.0 \%$ \\
\hline $\mathrm{H}_{2} \mathrm{~N}_{3}$ & 974.6 & {$[\mathrm{M}+\mathrm{Na}]^{+}$} & $0.0 \%$ & $0.0 \%$ & $1.3 \%$ & $1.2 \%$ & $0.0 \%$ & $0.0 \%$ & $0.0 \%$ & $0.0 \%$ \\
\hline $\mathrm{H}_{5} \mathrm{~N}_{3}$ & $1460.6 ; 742.1$ & {$[\mathrm{M}+\mathrm{Na}]^{+} ;[\mathrm{M}+2 \mathrm{Na}]^{2+}$} & $0.0 \%$ & $27.1 \%$ & $0.0 \%$ & $0.0 \%$ & $0.0 \%$ & $0.0 \%$ & $0.2 \%$ & $0.3 \%$ \\
\hline $\mathrm{H}_{6} \mathrm{~N}_{4}$ & 924.5 & {$[\mathrm{M}+\mathrm{Na}]^{+}$} & $0.0 \%$ & $3.0 \%$ & $0.0 \%$ & $0.0 \%$ & $0.0 \%$ & $0.0 \%$ & $0.0 \%$ & $0.0 \%$ \\
\hline $\mathrm{H}_{7} \mathrm{~N}_{5}$ & 1107.0 & {$[\mathrm{M}+2 \mathrm{Na}]^{2+}$} & $0.0 \%$ & $0.3 \%$ & $0.0 \%$ & $0.0 \%$ & $0.0 \%$ & $0.0 \%$ & $0.0 \%$ & $0.0 \%$ \\
\hline $\mathrm{HN}$ & 406.2 & {$[\mathrm{M}+\mathrm{Na}]^{+}$} & $1.4 \%$ & $1.9 \%$ & $3.6 \%$ & $2.5 \%$ & $0.0 \%$ & $0.0 \%$ & $0.0 \%$ & $5.4 \%$ \\
\hline $\mathrm{H}_{2} \mathrm{~N}$ & 568.4 & {$[\mathrm{M}+\mathrm{Na}]^{+}$} & $0.5 \%$ & $0.3 \%$ & $0.9 \%$ & $1.8 \%$ & $53.7 \%$ & $50.1 \%$ & $54.7 \%$ & $1.1 \%$ \\
\hline $\mathrm{H}_{3} \mathrm{~N}$ & 730.4 & {$[\mathrm{M}+\mathrm{Na}]^{+}$} & $0.0 \%$ & $0.5 \%$ & $0.4 \%$ & $0.5 \%$ & $14.1 \%$ & $13.9 \%$ & $15.2 \%$ & $1.3 \%$ \\
\hline $\mathrm{H}_{4} \mathrm{~N}$ & 892.5 & {$[\mathrm{M}+\mathrm{Na}]^{+}$} & $0.0 \%$ & $0.0 \%$ & $0.3 \%$ & $0.3 \%$ & $11.9 \%$ & $13.5 \%$ & $12.6 \%$ & $1.4 \%$ \\
\hline $\mathrm{H}_{5} \mathrm{~N}$ & 1054.5 & {$[\mathrm{M}+\mathrm{Na}]^{+}$} & $0.0 \%$ & $0.0 \%$ & $0.2 \%$ & $0.0 \%$ & $3.2 \%$ & $4.0 \%$ & $3.2 \%$ & $0.0 \%$ \\
\hline $\mathrm{H}_{6} \mathrm{~N}$ & 1216.5 & {$[\mathrm{M}+\mathrm{Na}]^{+}$} & $0.0 \%$ & $0.0 \%$ & $0.0 \%$ & $0.0 \%$ & $1.3 \%$ & $1.5 \%$ & $1.2 \%$ & $0.0 \%$ \\
\hline $\mathrm{H}_{7} \mathrm{~N}$ & $1378.5 ; 700.9$ & {$[\mathrm{M}+\mathrm{Na}]^{+} ;[\mathrm{M}+2 \mathrm{Na}]^{2+}$} & $0.0 \%$ & $0.0 \%$ & $0.0 \%$ & $0.0 \%$ & $0.6 \%$ & $0.7 \%$ & $0.5 \%$ & $0.0 \%$ \\
\hline $\mathrm{H}_{8} \mathrm{~N}$ & $1540.4 ; 782.0$ & {$[\mathrm{M}+\mathrm{Na}]^{+} ;[\mathrm{M}+2 \mathrm{Na}]^{2+}$} & $0.0 \%$ & $0.0 \%$ & $0.0 \%$ & $0.0 \%$ & $0.5 \%$ & $0.5 \%$ & $0.4 \%$ & $0.0 \%$ \\
\hline $\mathrm{H}_{9} \mathrm{~N}$ & $1702.8 ; 863.0$ & {$[\mathrm{M}+\mathrm{Na}]^{+} ;[\mathrm{M}+2 \mathrm{Na}]^{2+}$} & $0.0 \%$ & $0.0 \%$ & $0.0 \%$ & $0.0 \%$ & $0.2 \%$ & $0.2 \%$ & $0.3 \%$ & $0.0 \%$ \\
\hline HS & 516.3 & {$[\mathrm{M}-\mathrm{H}+2 \mathrm{Na}]^{+}$} & $0.8 \%$ & $0.0 \%$ & $0.2 \%$ & $0.4 \%$ & $0.1 \%$ & $0.1 \%$ & $0.0 \%$ & $1.0 \%$ \\
\hline $\mathrm{H}_{2} \mathrm{~S}$ & $678.5 ; 656.5$ & {$[\mathrm{M}-\mathrm{H}+2 \mathrm{Na}]^{+} ;[\mathrm{M}+\mathrm{Na}]^{+}$} & $2.7 \%$ & $1.5 \%$ & $2.3 \%$ & $2.3 \%$ & $0.7 \%$ & $0.5 \%$ & $0.6 \%$ & $2.2 \%$ \\
\hline NS & & & $0.0 \%$ & $0.0 \%$ & $0.0 \%$ & $0.0 \%$ & $0.0 \%$ & $0.0 \%$ & $0.0 \%$ & $0.0 \%$ \\
\hline HNS & $719.5 ; 697.6$ & {$[\mathrm{M}-\mathrm{H}+2 \mathrm{Na}]^{+} ;[\mathrm{M}+\mathrm{Na}]^{+}$} & $2.9 \%$ & $0.7 \%$ & $1.6 \%$ & $1.6 \%$ & $0.4 \%$ & $0.6 \%$ & $0.5 \%$ & $2.7 \%$ \\
\hline $\mathrm{N}_{2} \mathrm{~S}$ & $760.3 ; 738.4$ & {$[\mathrm{M}-\mathrm{H}+2 \mathrm{Na}]^{+} ;[\mathrm{M}+\mathrm{Na}]^{+}$} & $0.0 \%$ & $0.0 \%$ & $0.1 \%$ & $0.2 \%$ & $0.0 \%$ & $0.0 \%$ & $0.0 \%$ & $0.0 \%$ \\
\hline $\mathrm{H}_{3} \mathrm{~N}_{2} \mathrm{~S}$ & $1246.8 ; 635.1$ & {$[\mathrm{M}-\mathrm{H}+2 \mathrm{Na}]^{+} ;[\mathrm{M}-\mathrm{H}+3 \mathrm{Na}]^{2+}$} & $0.0 \%$ & $0.0 \%$ & $0.0 \%$ & $0.0 \%$ & $0.0 \%$ & $0.0 \%$ & $0.0 \%$ & $18.7 \%$ \\
\hline $\mathrm{H}_{5} \mathrm{~N}_{3} \mathrm{~S}$ & $898.3 ; 887.6$ & {$[\mathrm{M}-\mathrm{H}+3 \mathrm{Na}]^{2+} ;[\mathrm{M}+2 \mathrm{Na}]^{2+}$} & $0.0 \%$ & $0.2 \%$ & $0.0 \%$ & $0.0 \%$ & $0.0 \%$ & $0.0 \%$ & $0.0 \%$ & $3.2 \%$ \\
\hline $\mathrm{H}_{5} \mathrm{~N}_{3} \mathrm{~S}_{2}$ & $1055.1 ; 1044.1$ & {$[\mathrm{M}-2 \mathrm{H}+4 \mathrm{Na}]^{2+} ;[\mathrm{M}-\mathrm{H}+3 \mathrm{Na}]^{2+}$} & $0.0 \%$ & $0.0 \%$ & $0.0 \%$ & $0.0 \%$ & $0.0 \%$ & $0.0 \%$ & $0.0 \%$ & $8.7 \%$ \\
\hline $\mathrm{H}_{6} \mathrm{~N}_{4} \mathrm{~S}_{2}$ & 1237.4 & {$[\mathrm{M}-2 \mathrm{H}+4 \mathrm{Na}]^{2+}$} & $0.0 \%$ & $0.0 \%$ & $0.0 \%$ & $0.0 \%$ & $0.0 \%$ & $0.0 \%$ & $0.0 \%$ & $0.0 \%$ \\
\hline $\mathrm{H}_{6} \mathrm{~N}_{4} \mathrm{~S}_{3}$ & & & $0.0 \%$ & $0.0 \%$ & $0.0 \%$ & $0.0 \%$ & $0.0 \%$ & $0.0 \%$ & $0.0 \%$ & $0.0 \%$ \\
\hline $\mathrm{H}_{7} \mathrm{~N}_{5} \mathrm{~S}_{2}$ & & & $0.0 \%$ & $0.0 \%$ & $0.0 \%$ & $0.0 \%$ & $0.0 \%$ & $0.0 \%$ & $0.0 \%$ & $0.0 \%$ \\
\hline $\mathrm{H}_{7} \mathrm{~N}_{5} \mathrm{~S}_{3}$ & & & $0.0 \%$ & $0.0 \%$ & $0.0 \%$ & $0.0 \%$ & $0.0 \%$ & $0.0 \%$ & $0.0 \%$ & $0.0 \%$ \\
\hline $\mathrm{H}_{3}\left(\mathrm{SO}_{3}\right) \mathrm{N}_{2} \mathrm{~S}$ & $1348.2 ; 674.5$ & {$[\mathrm{M}-2 \mathrm{H}+3 \mathrm{Na}]^{+} ;[\mathrm{M}-\mathrm{H}+3 \mathrm{Na}]^{2+}$} & $0.0 \%$ & $0.0 \%$ & $0.0 \%$ & $0.0 \%$ & $0.0 \%$ & $0.0 \%$ & $0.0 \%$ & $2.4 \%$ \\
\hline $\mathrm{H}_{5}\left(\mathrm{SO}_{3}\right) \mathrm{N}_{3} \mathrm{~S}$ & $1854.3 ; 949.4$ & {$[\mathrm{M}-\mathrm{H}+2 \mathrm{Na}]^{+} ;[\mathrm{M}-2 \mathrm{H}+4 \mathrm{Na}]^{2+}$} & $0.0 \%$ & $0.0 \%$ & $0.0 \%$ & $0.0 \%$ & $0.0 \%$ & $0.0 \%$ & $0.0 \%$ & $0.9 \%$ \\
\hline $\mathrm{H}_{5}\left(\mathrm{SO}_{3}\right) \mathrm{N}_{3} \mathrm{~S}_{2}$ & $2166.6 ; 1083.8$ & {$[\mathrm{M}-2 \mathrm{H}+3 \mathrm{Na}]^{+} ;[\mathrm{M}-\mathrm{H}+3 \mathrm{Na}]^{2+}$} & $0.0 \%$ & $0.0 \%$ & $0.0 \%$ & $0.0 \%$ & $0.0 \%$ & $0.0 \%$ & $0.0 \%$ & $1.0 \%$ \\
\hline $\mathrm{H}_{6}\left(\mathrm{SO}_{3}\right) \mathrm{N}_{4} \mathrm{~S}_{3}$ & & & $0.0 \%$ & $0.0 \%$ & $0.0 \%$ & $0.0 \%$ & $0.0 \%$ & $0.0 \%$ & $0.0 \%$ & $0.0 \%$ \\
\hline $\mathrm{H}_{2} \mathrm{NS}$ & & & $0.0 \%$ & $0.0 \%$ & $0.0 \%$ & $0.0 \%$ & $0.0 \%$ & $0.0 \%$ & $0.0 \%$ & $0.0 \%$ \\
\hline $\mathrm{S}_{2}$ & & & $0.0 \%$ & $0.0 \%$ & $0.0 \%$ & $0.0 \%$ & $0.0 \%$ & $0.0 \%$ & $0.0 \%$ & $0.0 \%$ \\
\hline
\end{tabular}


Table 2 (continued)

\begin{tabular}{|c|c|c|c|c|c|c|c|c|c|c|}
\hline \multirow[t]{3}{*}{ Comp. } & \multirow{3}{*}{$\begin{array}{l}\text { Registered } \\
m / z\end{array}$} & \multirow[t]{3}{*}{ Charge state } & \multicolumn{8}{|c|}{ Relative area } \\
\hline & & & \multirow{2}{*}{$\begin{array}{l}\text { Fuc. } \\
\text { U1 }\end{array}$} & \multirow{2}{*}{$\begin{array}{l}\mathrm{G}_{\mathrm{M} 1} \text {-gang. } \\
\mathrm{U} 2\end{array}$} & \multicolumn{2}{|c|}{$\mathrm{G}_{\mathrm{M} 2}$-gangliosidosis } & \multicolumn{3}{|c|}{$\alpha$-Mannosidosis } & \multirow{2}{*}{$\begin{array}{l}\text { Sial. } \\
\text { U8 }\end{array}$} \\
\hline & & & & & U3 & U4 & U5 & U6 & U7 & \\
\hline HX & & & $0.0 \%$ & $0.0 \%$ & $0.0 \%$ & $0.0 \%$ & $0.0 \%$ & $0.0 \%$ & $0.0 \%$ & $0.0 \%$ \\
\hline $\mathrm{H}_{2} \mathrm{X}$ & & & $0.0 \%$ & $0.0 \%$ & $0.0 \%$ & $0.0 \%$ & $0.0 \%$ & $0.0 \%$ & $0.0 \%$ & $0.0 \%$ \\
\hline HNX & $606.5 ; 584.3$ & {$[\mathrm{M}-\mathrm{H}+2 \mathrm{Na}]^{+} ;[\mathrm{M}+\mathrm{Na}]^{+}$} & $0.0 \%$ & $0.5 \%$ & $0.0 \%$ & $0.0 \%$ & $0.0 \%$ & $0.0 \%$ & $0.0 \%$ & $0.0 \%$ \\
\hline HNSX & & & $0.0 \%$ & $0.0 \%$ & $0.0 \%$ & $0.0 \%$ & $0.0 \%$ & $0.0 \%$ & $0.0 \%$ & $0.0 \%$ \\
\hline SX & & & $0.0 \%$ & $0.0 \%$ & $0.0 \%$ & $0.0 \%$ & $0.0 \%$ & $0.0 \%$ & $0.0 \%$ & $0.0 \%$ \\
\hline $\mathrm{N}_{2} \mathrm{NeuGc}$ & & & $0.0 \%$ & $0.0 \%$ & $0.0 \%$ & $0.0 \%$ & $0.0 \%$ & $0.0 \%$ & $0.0 \%$ & $0.0 \%$ \\
\hline $\mathrm{H}_{2}$ & 365.2 & {$[\mathrm{M}+\mathrm{Na}]^{+}$} & $48.7 \%$ & $35.8 \%$ & $46.7 \%$ & $32.4 \%$ & $7.1 \%$ & $4.9 \%$ & $3.2 \%$ & $26.6 \%$ \\
\hline $\mathrm{H}_{3}$ & 527.3 & {$[\mathrm{M}+\mathrm{Na}]^{+}$} & $5.2 \%$ & $3.8 \%$ & $6.2 \%$ & $5.6 \%$ & $0.9 \%$ & $2.9 \%$ & $1.1 \%$ & $6.5 \%$ \\
\hline $\mathrm{H}_{4}$ & 689.5 & {$[\mathrm{M}+\mathrm{Na}]^{+}$} & $1.6 \%$ & $0.5 \%$ & $2.2 \%$ & $0.7 \%$ & $0.4 \%$ & $1.0 \%$ & $0.8 \%$ & $2.8 \%$ \\
\hline
\end{tabular}

Comp composition, Fuc Fucosidosis, GM1-gang $\mathrm{G}_{\mathrm{M} 1}$-gangliosidosis, Sial sialidosis

detected MS signals. Glycan species were characterized by tandem mass spectrometry as exemplified for the fucosyl disaccharide Fuc-HexNAc which had a relative abundance of $10.6 \%$ (Table 2; Fig. 2). The MS/MS fragmentation spectrum (Fig. 2) showed $Z_{1}$ and $B_{1}$ fragments as well as a prominent signal arising from the loss of water $(\mathrm{m} / \mathrm{z} 372.1)$. Cross-ring cleavages at $\mathrm{m} / z 229.0\left({ }^{0,4} \mathrm{~A}_{2}\right), 259.0\left({ }^{0,3} \mathrm{~A}_{2}\right)$, and $289.0\left({ }^{0,2} \mathrm{~A}_{2}\right)$ suggest a $1-6$ linkage for the fucose residue [41-43]. From the total set of 54 glycan compositions observed in this study, 17 were found to be present in the fucosidosis sample (see Electronic supplementary material Table S1) resulting in a glycan fingerprint as shown in Fig. 3a. Of the eight fucosylated oligosaccharides detected, three have already been previously shown to be related to fucosidosis [2, 29, 30, 37, 44].

\section{Urinary glycans in $\alpha$-mannosidosis}

Three urine samples of three different $\alpha$-mannosidosis patients from two different families were analyzed. In all three samples, 17 endo- $\beta-N$-acetylglucosaminidase cleavage products of mannose-rich oligosaccharides of composition $\mathrm{Hex}_{2-9} \mathrm{Hex}$ NAc were detected [33, 45] (Table 2, Electronic supplementary material S2). The proposed structures are derived from literature $[32,33,45]$ as well as from the obtained tandem MS data. An example of a fragment ion spectrum of the major $\mathrm{Hex}_{3} \mathrm{Hex}-$
Fig. 2 Positive-ion fragmentation mass spectrum of the monosodiated disaccharide $\mathrm{HexNAc}_{1} \mathrm{Fuc}_{1}$ (precursor ion at $\mathrm{m} / \mathrm{z}$ 390.2) from urine of a fucosidosis patient. Red triangle $=$ fucose, blue square $=$ $N$-acetylglucosamine

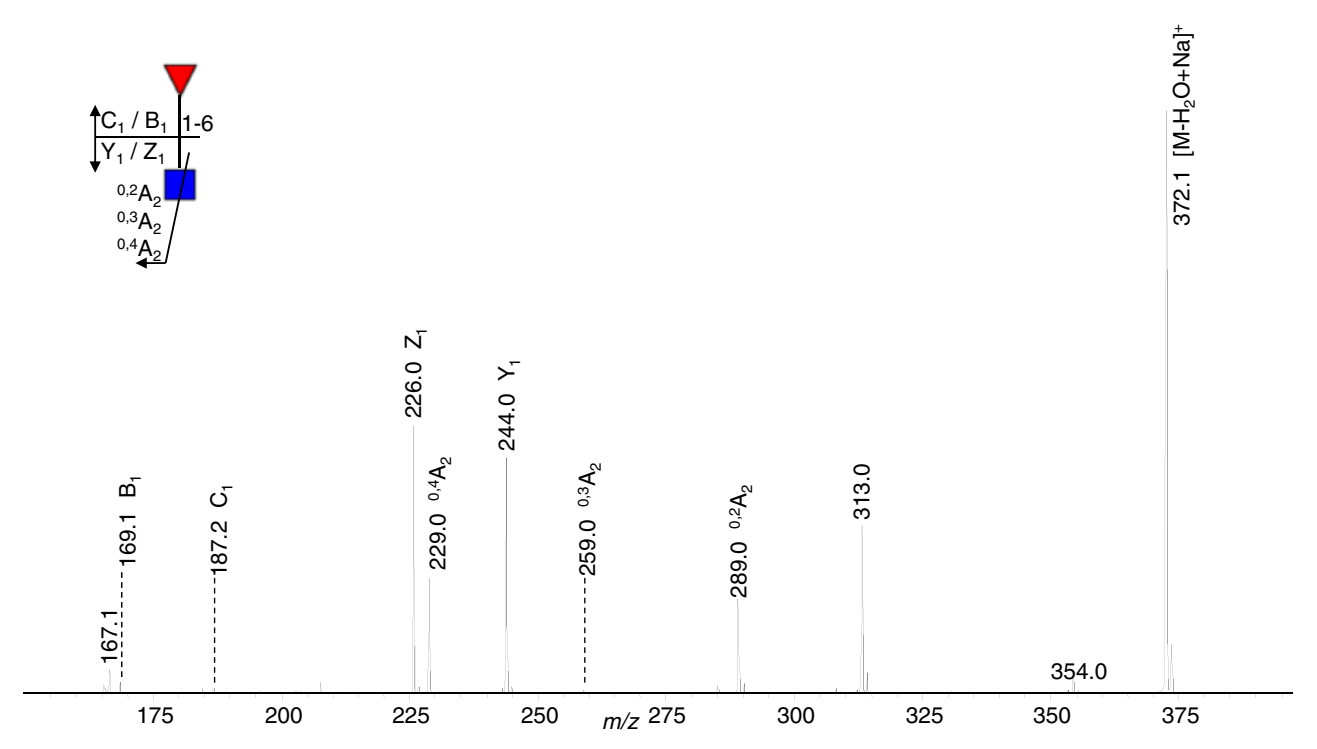



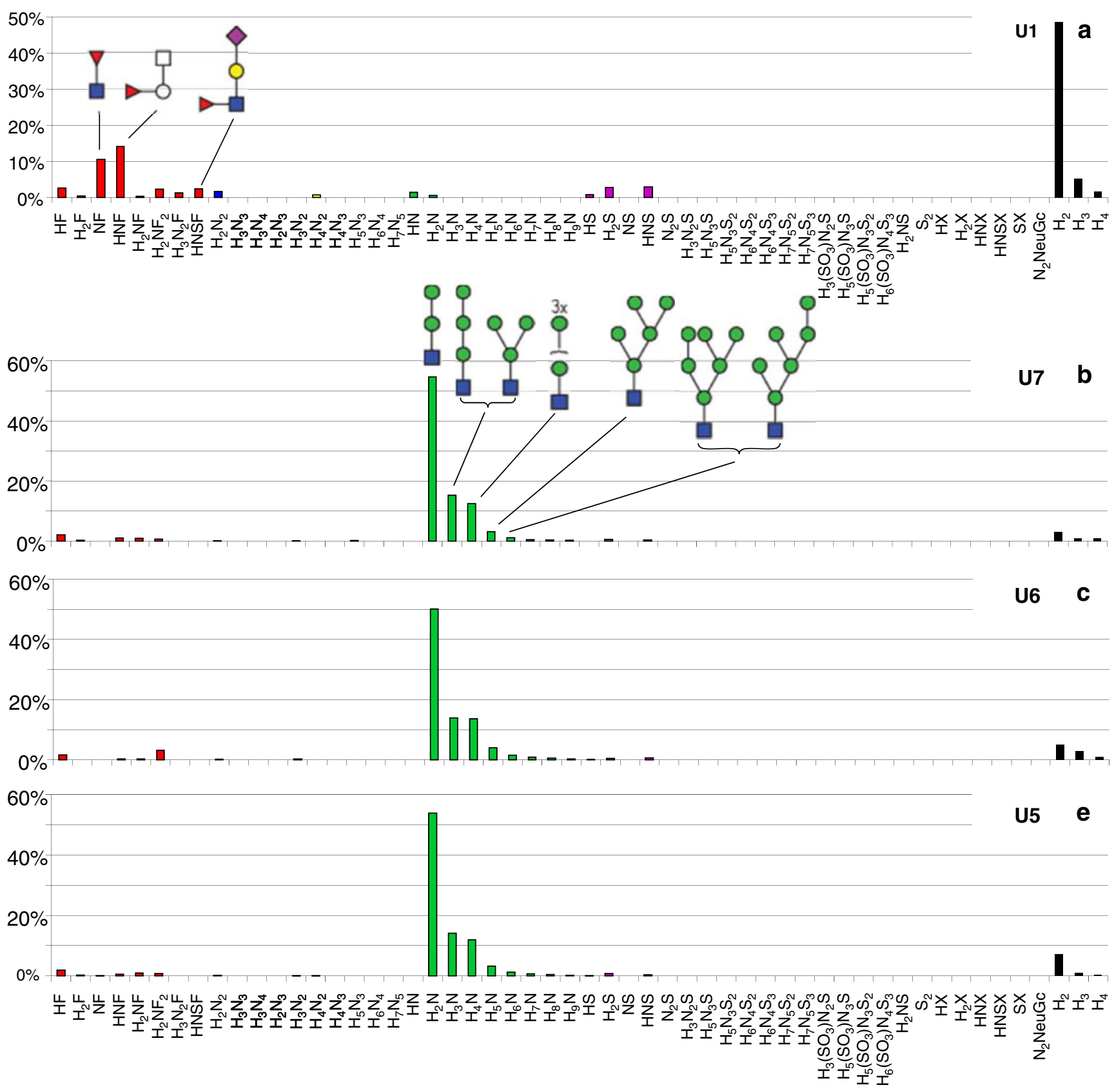

Fig. 3 Histograms showing the relative abundance of the detected glycans in the urine sample (Table 1) of lysosomal storage disorders fucosidosis (a), $\alpha$-mannosidosis (b, c, and d), $\mathrm{G}_{\mathrm{M} 1}$-gangliosidosis (e), $\mathrm{G}_{\mathrm{M}^{-}}$ gangliosidosis (f and $\mathbf{g}$ ), and sialidosis (h). $H$ or white circle $=$ hexose, $N$ or

$\mathrm{NAc}_{1}$ isomer is shown in Fig. 4. The ${ }^{0,2} \mathrm{~A}_{3}$ and ${ }^{2,4} \mathrm{~A}_{3}$ ions are typical for a 4-substituted HexNAc at the reducing end [41-43]. The cross-ring fragment ${ }^{0,3} \mathrm{~A}_{2}(\mathrm{~m} / z$ 275.2) is indicative for a 6substituted hexose [41-43]. The $\mathrm{B}_{2} \mathrm{Y}_{2 \alpha}$ ion (D-ion, $m / z$ 347.3) reveals the composition of the 6-antenna [42]. Histograms giving the relative abundances of the observed glycans are shown in Fig. 3b-d. The three urine samples resulted in very similar profiles including a prominent signal corresponding to white square $=N$-acetylhexosamine, $F$ or red triangle $=$ fucose, $S$ or purple diamond $=N$-acetylneuraminic acid, $\mathrm{NeuGc}=N$-glycolylneuraminic acid, $X=$ hexonic acid, $\mathrm{SO}_{3}=$ sulfate, yellow circle $=$ galactose, green circle $=$ mannose, blue square $=N$-acetylglucosamine

$\mathrm{Hex}_{2} \mathrm{HexNAc}_{1}$. The whole set of oligomannosidic structures was detected $\left(\mathrm{Hex}_{2-9} \mathrm{HexNAc}_{1}\right)$ showing decreasing signals with increasing size.

Urinary glycans in $\mathrm{G}_{\mathrm{M} 1}$-gangliosidosis

Extracted ion chromatograms of the disease-related glycans found in the urine of a $\mathrm{G}_{\mathrm{M} 1}$-gangliosidosis patient are 


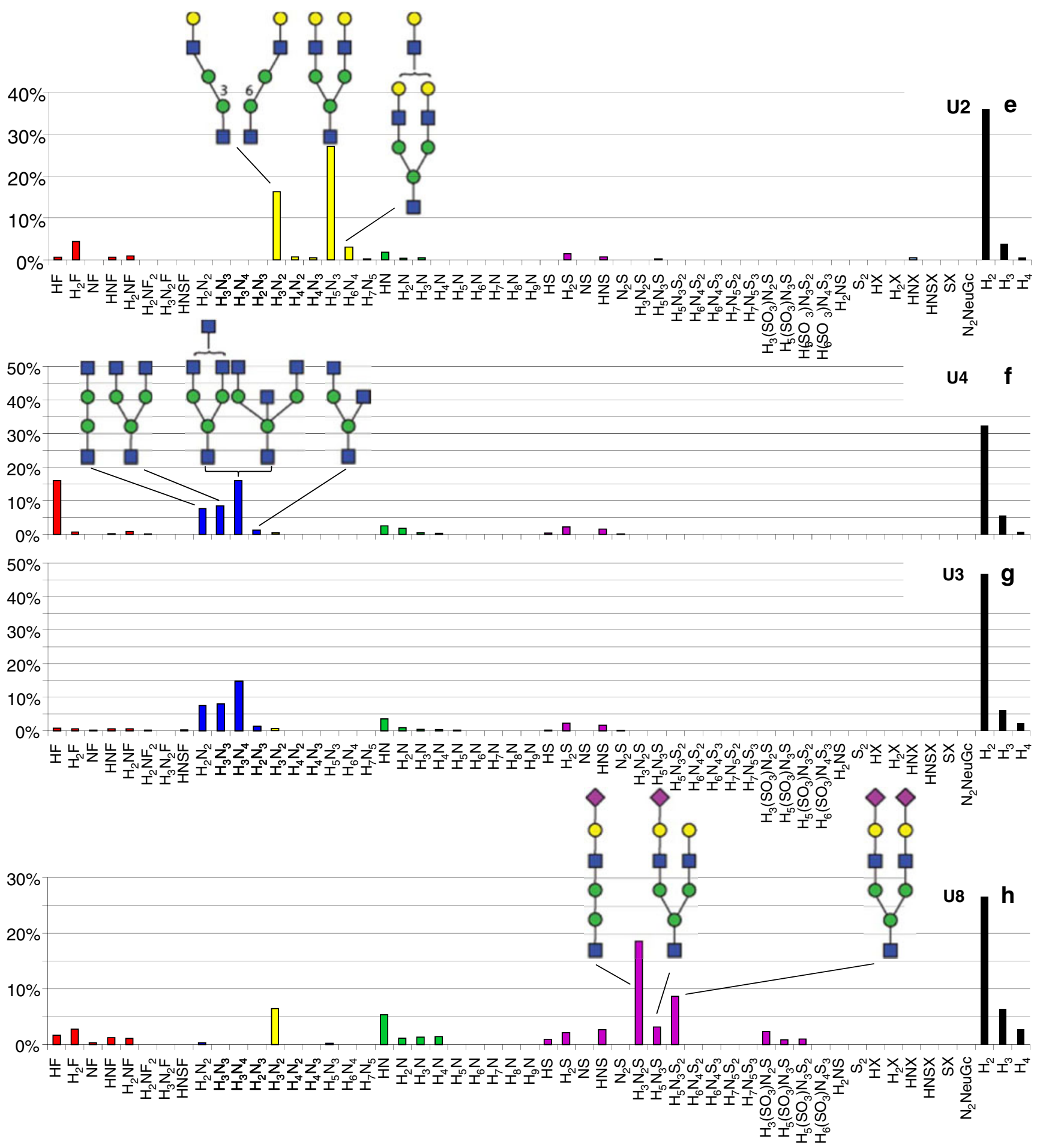

Fig. 3 (continued)

represented in Fig. 5. Twenty glycan compositions were detected, and six of those structures with the composition $\mathrm{Hex}_{3-7} \mathrm{HexNAc}_{2-5}$ are presumably disease-related (Table 2, Electronic supplementary material Table S3). The compositions as well as the tandem mass spectrometric data (see Electronic supplementary material Table S3) suggest these glycans to be endo- $\beta-N$-acetylglucosaminidase cleavage products of complex type $\mathrm{N}$-glycans. Composition $\mathrm{Hex}_{3} \mathrm{Hex}$ $\mathrm{NAc}_{2}$ was interpreted as monoantennary and $\mathrm{Hex}_{5} \mathrm{HexNAc}_{3}$ as diantennary structure. Species carrying additional $\mathrm{Hex}_{1}$. $\mathrm{HexNAc}_{1}$ units were found to be attached resulting in $\mathrm{Hex}_{6-}$ $\mathrm{HexNAc}_{4}$ and $\mathrm{Hex}_{7} \mathrm{HexNAc}_{5}$ species carrying more antennae 


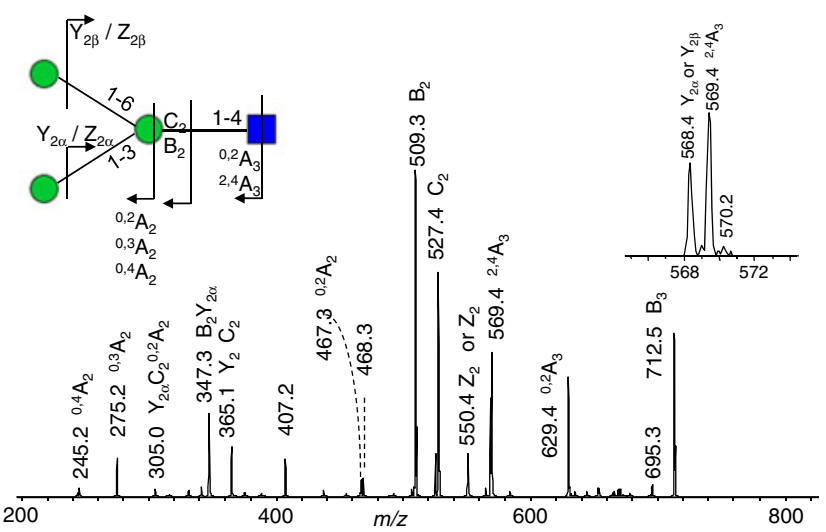

Fig. 4 Positive-ion fragmentation mass spectrum of the monosodiated tetrasaccharide $\mathrm{Man}_{3} \mathrm{GlcNAc}_{1}$ (precursor ion at $\mathrm{m} / \mathrm{z}$ 730.6) from urine of a $\alpha$-mannosidosis patient. Green circle $=$ mannose, blue square $=N$ acetylglucosamine

as well as LacNAc repeats $[9,34,35]$. In addition, a relatively low amount of a trisaccharide with the composition $\mathrm{Hex}_{1} \mathrm{Hex}-$ $\mathrm{NAc}_{1} \mathrm{HexonA}_{1}$ was detected (Table 2). A histogram showing the relative abundance of the observed glycans is given in Fig. 3e with high signals corresponding to $\mathrm{Hex}_{3} \mathrm{HexNAc}_{2}$ and $\mathrm{Hex}_{5} \mathrm{HexNAc}_{3}$. The tandem MS spectrum of the disodiated diantennary $N$-glycan with the composition $\mathrm{Hex}_{5} \mathrm{HexNAc}_{3}(\mathrm{~m} /$ $z$ 742.1) is shown in Fig. 6. The cross-ring fragments ${ }^{0,2} \mathrm{~A}_{5}$ and ${ }^{2,4} \mathrm{~A}_{5}$ are typical for a 4 -substituted reducing end HexNAc

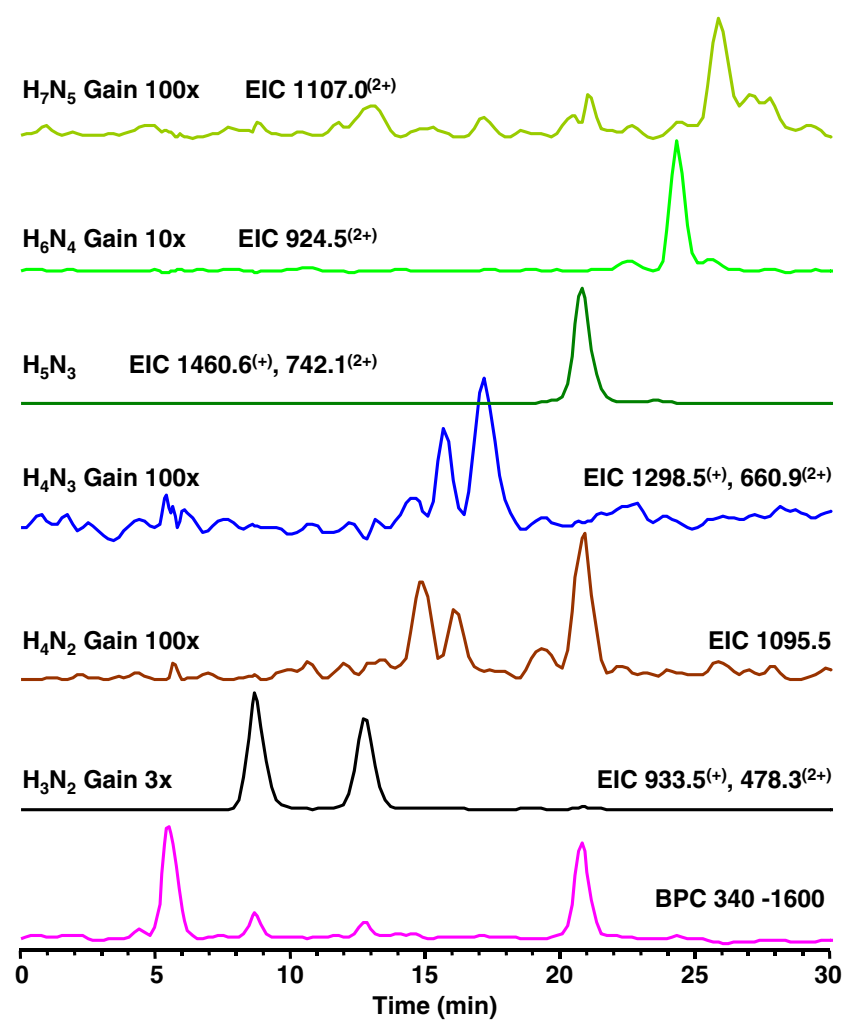

Fig. 5 Separation of oligosaccharides in urine of a $\mathrm{G}_{\mathrm{M} 1}$-gangliosidosis patient. $H$ hexose, $N N$-acetylhexosamine, $B P C$ base peak chromatogram
[41-43]. The fragment ion $\mathrm{B}_{4} \mathrm{Y}_{2 \alpha}$ (D-ion, $m / z$ 712.3) reveals the composition of the 6-antenna [42]. The characterization of the two well-separated isomers with the composition $\mathrm{Hex}_{3} \mathrm{Hex}$ $\mathrm{NAc}_{2}$ has been reported earlier [25].

Urinary glycans in $\mathrm{G}_{\mathrm{M} 2}$-gangliosidosis

The analysis of the urine samples of two $\mathrm{G}_{\mathrm{M} 2}$-gangliosidosis patients revealed $11 \mathrm{G}_{\mathrm{M} 2}$-gangliosidosis-related glycan isomers (Table 2, Electronic supplementary material Table S4) with the composition $\mathrm{Hex}_{2-3} \mathrm{HexNAc}_{2-4}$. The proposed structures reported in Electronic supplementary material Table S4 are based on our MS/MS results and on the known urinary oligosaccharides related to $\beta$-hexosaminidase deficiency in $\mathrm{G}_{\mathrm{M}^{-}}$ gangliosidos $[15,16]$. $\mathrm{Hex}_{2} \mathrm{HexNAc}_{2}$ corresponds to a monoantennary, $\mathrm{Hex}_{2} \mathrm{HexNAc}_{3}$ to a bisected monoantennary, $\mathrm{Hex}_{3-}$ $\mathrm{HexNAc}_{3}$ to a diantennary, and $\mathrm{Hex}_{3} \mathrm{HexNAc}_{4}$ to a triantennary or bisected diantennary structure [16]. Figure 7 shows an excellent example of the isomeric separation of the two reported monoantennary glycans $\left(\mathrm{Hex}_{2} \mathrm{HexNAc}_{2} ; \mathrm{m} / \mathrm{z}\right.$ 771.5). The EIC corresponding to $\mathrm{Hex}_{2} \mathrm{HexNAc}_{2}(\mathrm{~m} / z$ 771.5) shows the separation of these isobaric structures (retention times 10.3 and 11.9 min; Fig. 7), and the MS/MS spectra are shown in Fig. 8. The observed series of B-ions are in accordance with a monosaccharide sequence of HexNAc-Hex-HexHexNAc for both isomers. Both oligosaccharides contain $N$ acetylhexosamine at the reducing end, which shows the crossring fragments ${ }^{0,2} \mathrm{~A}_{4}(m / z 670)$ and ${ }^{2,4} \mathrm{~A}_{4}(m / z 610)$ indicative for a 4-substituted $\mathrm{N}$-acetylhexosamine [41-43]. The observed cross-ring fragments ${ }^{0,2} \mathrm{~A}_{3}(m / z 508),{ }^{0,3} \mathrm{~A}_{3}(m / z 478)$, and ${ }^{0,4} \mathrm{~A}_{3}$ $(\mathrm{m} / \mathrm{z} 448)$ observed for the adjacent hexose are typical for a 6-substitution. Based on the observed MS/MS data and literature data [15], glycan A was identified as the $\mathrm{G}_{\mathrm{M} 2}$-gangliosidosis urinary tetrasaccharide $\operatorname{GlcNAc}(\beta 1-2) \operatorname{Man}(\alpha 1-6)$ $\operatorname{Man}(\beta 1-4)$ GlcNAc. A lack of $A_{3}$ cross-ring fragments, which is typical for a 3 -substituted sugar, indicates that glycan $B$ is the isomer $\operatorname{GlcNAc}(\beta 1-2) \operatorname{Man}(\alpha 1-3) \operatorname{Man}(\beta 1-4)$ GlcNAc. Hence, the linkage-specific fragmentation allowed the assignment of the observed glycans to two urinary glycans related to

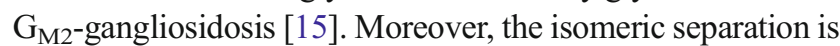
emphasized by the different elution times of the three isomers corresponding to $\mathrm{Hex}_{3} \mathrm{HexNAc}_{3}$ registered in monosodidated $(\mathrm{m} / \mathrm{z} 1136.5)$ as well as disodiated $(\mathrm{m} / \mathrm{z} 580.0)$ form. Figure $3 \mathrm{f}$ and $g$ show the relative abundance of the detected glycans for the two urine samples. In both samples, a high relative abundance of $\mathrm{Hex}_{2} \mathrm{HexNAc}_{2}$ (both samples 7.6\%), $\mathrm{Hex}_{2} \mathrm{HexNAc}_{3}$ $(1.3 \%$ and $1.2 \%), \mathrm{Hex}_{3} \mathrm{HexNAc}_{3}(8.0 \%$ and $8.2 \%)$, and $\mathrm{Hex}_{3-}$ $\mathrm{HexNAc}_{4}$ (14.8\% and 15.5\%) was observed.

Urinary glycans in sialidosis

Analysis of the urine of a sialidosis patient revealed eight disease-related sialylated oligosaccharides (Table 2, Electronic 
Fig. 6 Positive-ion

fragmentation mass spectrum of the disodiated diantennary oligosaccharide $\mathrm{Hex}_{5} \mathrm{HexNAc}_{3}$ (precursor ion at $\mathrm{m} / \mathrm{z} 742.1$ )

from urine of a $\mathrm{G}_{\mathrm{M} 1^{-}}$

gangliosidosis patient.

Yellow circle galactose, green

circle mannose, blue

square $\mathrm{N}$-acetylglucosamine

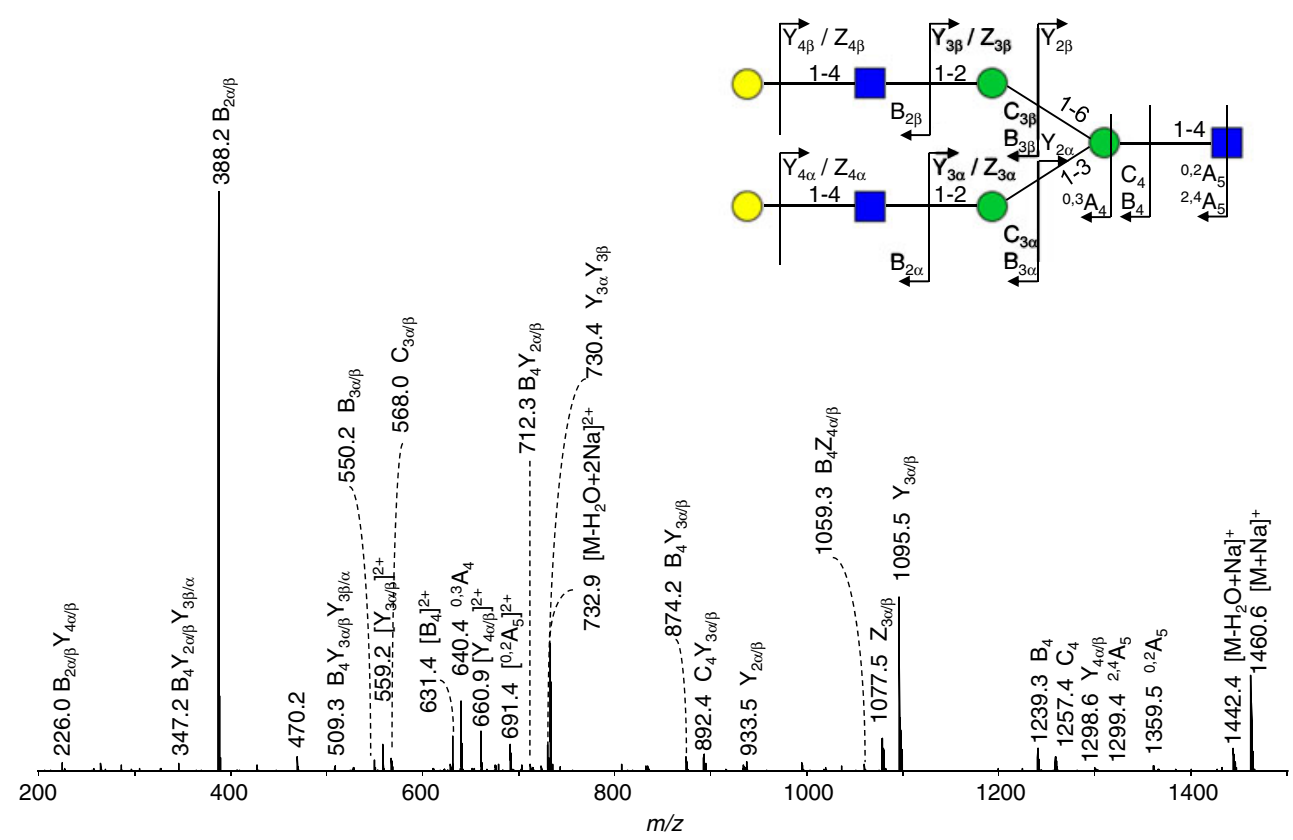

supplementary material S5). Structures with a high relative abundance such as $\mathrm{Hex}_{3} \mathrm{HexNAc}_{2}(6.5 \%)$, HexHexNAc (5.4\%), $\mathrm{Hex}_{3} \mathrm{HexNAc}_{2} \mathrm{Neu} 5 \mathrm{Ac}(18.7 \%)$, and $\mathrm{Hex}_{5} \mathrm{Hex}-$ $\mathrm{NAc}_{3} \mathrm{Neu} 5 \mathrm{Ac}_{2}(8.7 \%)$ were detected (Fig. 3h). The presence of sulfated sialyloligosaccharides $\mathrm{H}_{3-5} \mathrm{SO}_{3} \mathrm{~N}_{2-3} \mathrm{~S}_{1-2}$ is noteworthy [26].

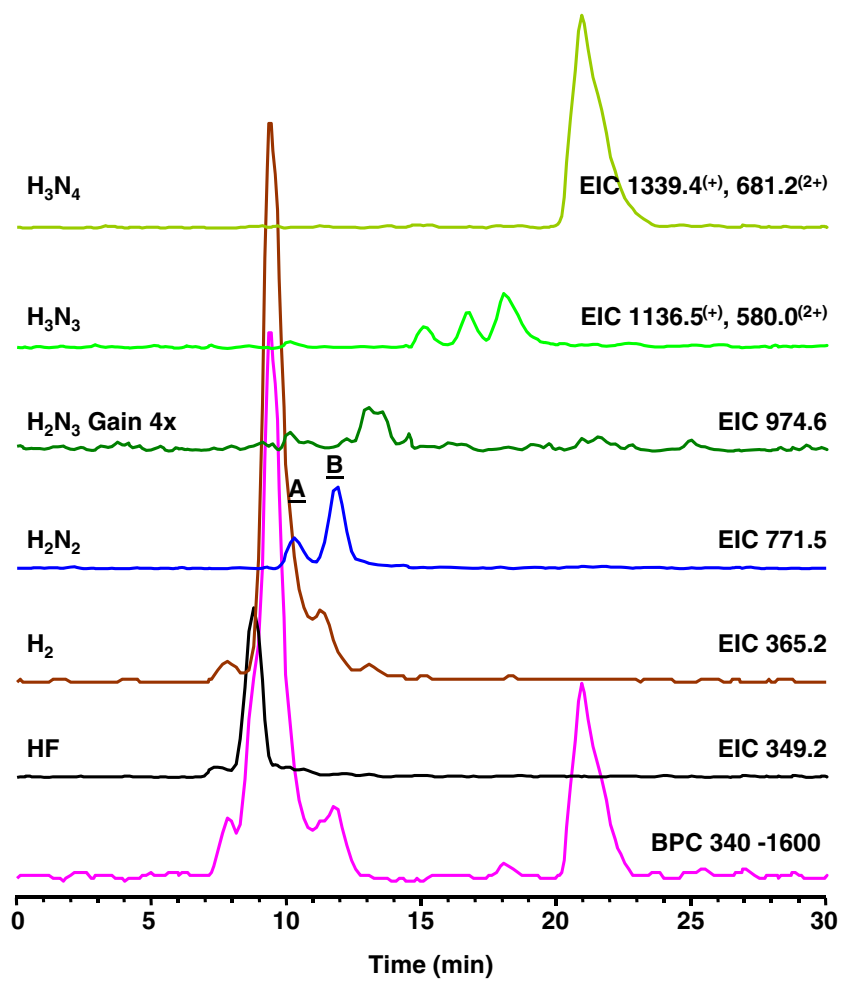

Fig. 7 Separation of oligosaccharides in urine of a $\mathrm{G}_{\mathrm{M} 2}$-gangliosisis patient. Fragment ion spectra of the species A and B is shown in Fig. 8. $F$ fucose, $H$ hexose, $N N$-acetylhexosamine, $B P C$ base peak chromatogram

\section{Discussion}

Using a prototype capillary HPAEC-IPAD-MS system for analyzing a set of 54 glycans in eight urine samples from patients with lysosomal storage disorders such as fucosidosis, $\alpha$-mannosidosis, $\mathrm{G}_{\mathrm{M} 1}$-gangliosidosis, $\mathrm{G}_{\mathrm{M} 2}$-gangliosidosis, and sialidosis (Table 1), we were able to find disease-related glycan structures. In addition, we identified glycan structures that are most probably diet- (human milk) or blood-grouprelated and are not related to the investigated disorders [37, 46-51] (see Table 2 and Electronic supplementary material Tables S1-S5). All urine samples, except for those of the mannosidosis patients, were found to contain a high relative amount of dihexose which is most likely a dietary product [37, $46,47]$. The presence of dietary products in urine is not surprising. We reported in a previous research about free oligosaccharides such as lactose, sialylhexose, and sialyllactose that we detected as major abundant carbohydrates in control urine samples of four healthy individuals [26].

Detection was performed using an ion trap mass spectrometer which was operated in automatic tandem MS mode resulting in informative fragment ion spectra for many glycans. Linkagespecific fragment ions [41-43] together with the known structural selectivity of high-performance anion-exchange chromatography [52-54] and literature knowledge on urinary oligosaccharides of LSDs [30, 55] made it possible to assign structures to most of the observed chromatographic signals.

Literature on fucosidosis reports that fucosylglycoasparagines are the most abundant glycoconjugates found in the urine of these patients $[2,56]$. These glycoconjugates are not expected to show up in our analysis, as they will presumably adsorb to or pass through the membrane of the online desalter. This phenomenon is due to the high negative charge density of 
Fig. 8 Positive-ion fragmentation mass spectra of two isomeric monosodiated tetrasaccharides $\mathrm{Hex}_{2} \mathrm{HexNAc}_{2}$ (precursor ion at $\mathrm{m} / \mathrm{z} 771.5$ ) from urine of a $\mathrm{G}_{\mathrm{M}^{-}}$ gangliosisis patient. The separation of A and B is shown in Fig. 7. Green circle mannose, blue square $\mathrm{N}$-acetylglucosamine

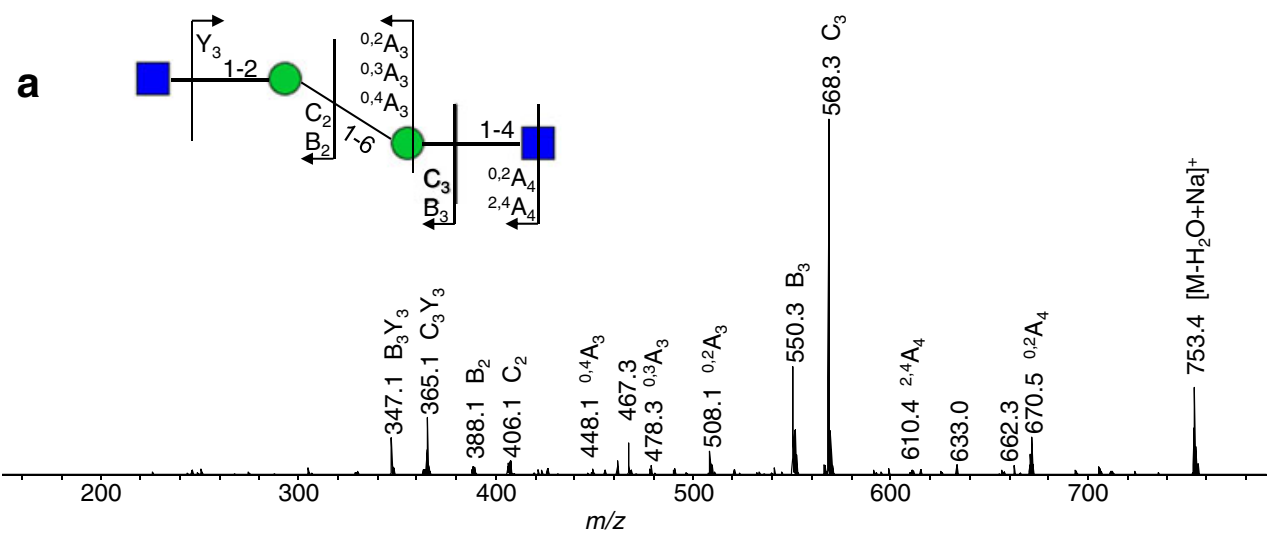

b
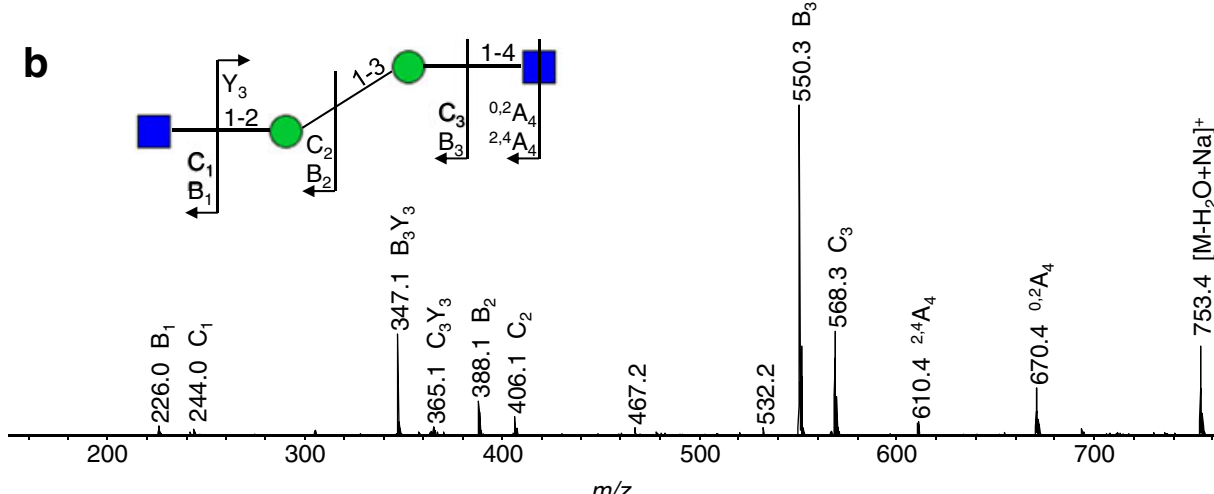

$m / z$

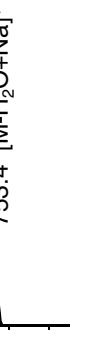

the fiber wall which is expected to result in strong interactions with cations such as glycopeptides entering the desalter [25]. Instead, we detected free fucosylsaccharides in the urine samples. The most abundant one is the disaccharide $\operatorname{Fuc}(\alpha 1-6)$ GlcNAc (Table 2, Electronic supplementary material Table S1, Figs. 2 and 3a) which is characteristic for this disorder [2, 56, 57]. Moreover, a trisaccharide with composition $\mathrm{Hex}_{1} \mathrm{HexNAc}_{1} \mathrm{Fuc}_{1}$ was found for fucosidosis (Fig. 3a). This trisaccharide is possibly the previously reported GalNAc $(\alpha 1-3)[$ Fuc $\alpha 1-2)]$ Gal [56]. In addition, Tsay et al. [58] and Nishigaki et al. [59] reported the presence of a fucosylated decasaccharide, however, this structure has neither been detected by us nor by Strecker et al. [56].

Moreover, 17 endo- $\beta-N$-acetylglucosaminidase cleavage products including chromatographically separated isomers were detected in all three urine samples of patients suffering from $\alpha$-mannosidosis (see Electronic supplementary material Table S2). These findings are in agreement with previous results reported by Matsuura et al. [33]. The authors identified in their study a similar number of endo- $\beta-N$-acetylglucosaminidase cleavage products [33]. However, while we observed three $\mathrm{Hex}_{4} \mathrm{HexNAc}_{1}$ isomers, three $\mathrm{Hex}_{5} \mathrm{HexNAc}_{1}$ isomers, and one $\mathrm{Hex}_{7} \mathrm{HexNAc}_{1}$ isomer, these authors found two, two, and three isomers, respectively (see Electronic supplementary material Table S2).

One of the three isomers of $\mathrm{Hex}_{3} \mathrm{HexNAc}_{3}$ found in the urine samples from patients suffering from $\mathrm{G}_{\mathrm{M} 2}$ gangliosidosis is in accordance with the diantennary structure with the core trimannose previously described by Strecker et al. [15, 60] while the other two isomers are probably monoantennary structures decorated with a $\operatorname{GlcNAc}(\beta 1-3) \mathrm{Gal}(\beta 1-4) \mathrm{GlcNAc}$ $(\beta 1-2)$ antenna. In the current study, we reported eight glycan structures that are related to sialidosis (see Electronic supplementary material Table S5). Of these eight glycans, five have been previously identified in sialidosis [39, 40, 50, 61]. We interpreted the structure of the glycan with the composition $\mathrm{Hex}_{1} \mathrm{HexNAc}_{1} \mathrm{Neu} 5 \mathrm{Ac}_{1}$ as Neu5Ac( $\left.\alpha 2-3 / 6\right) \mathrm{Gal}(\beta 1-4)$ GlcNAc and being related to sialidosis [50], although, based on our data, we cannot exclude that $\operatorname{Neu} 5 \operatorname{Ac}(\alpha 2-3 / 6) \mathrm{Gal}$ $(\beta 1-4)$ GlcNAc might be sialyllactosamine from milk [37, 62, 63]. All sialidosis-relevant carbohydrate structures described here are terminated with sialic acid residues, in accordance with the primary defect in exo- $\alpha$-sialidase. In addition, three $O$-sulfated oligosaccharides with terminal sialic acid residues were detected in the urine sample (Table 2) showing structures previously detected by us in galactosialidosis [26]. This may imply that the $O$-sulfated carbohydrates reported here are indeed related to the exo- $\alpha$-sialidase deficiency found in both galactosialidosis and sialidosis. MS detection in the positive ion mode is known to be less sensitive for negatively charged glycans such as sialyl- and $O$-sulfated-oligosaccharides. Therefore, this sample has also been analyzed in the negative ion mode and indeed more sialylated-, $O$-sulfated-glycans, and glycans having reducing end aldohexonic acid residue 
were observed due to the improved sensitivity for the detection of negatively charged molecules (data not shown).

All together, this publication shows the value of capillary HPAEC-IPAD-MS for analyzing oligosaccharides in clinical urine samples without the need for derivatization. This prototype analytical system features femtomol sensitivity for both pulsed amperometric detection and mass spectrometric detection [25] allowing the relatively low abundant $O$-sulfated-glycan moieties to be detected. In contrast to other liquid chromatography methods relying on reducing end labeling for detection and/or separation [64, 65], HPAEC-IPAD as well as HPAEC-MS do not depend on glycan labeling. Consequently, we were able to detect an oxidized oligosaccharide with an innermost aldohexonic acid residue in the $\mathrm{G}_{\mathrm{M} 1}$-ganglioside urine sample. Moreover, the setup used in this study enables the separation of isomeric glycans. Based on the efficient fragment ion analysis using an ion trap instrument, informative fragment spectra of sodium adducts can be obtained with minute amounts of material, allowing insights into defects of glycoconjugates degradation, and investigation of metabolic and catabolic pathways.

Although the used instrumentation is a prototype, similar analyses can be performed using commercially available narrow bore ion chromatographs [66], with presumably less sensitivity due to the bigger dimensions and higher flow rates. The desalter in such a system is based on a flat semipermeable cation exchange membrane and regenerated by electrolysis of water $[67,68]$.

LC-MS in general and the here described method in particular are analytically powerful. In the current research paper, we demonstrated that the HPAEC-MS technology in combination with MS/MS information on structural isomers is suitable for determining characteristic glycan fingerprints in lysosomal storage diseases which may have diagnostic potential.

Acknowledgment We would like to thank Dr. André Klein, Laboratoire de Biochimie et de Biologie Moléculaire, UAM de Glycopathologies, Centre de Biologie et Pathologie, Lille, France, for kindly providing a urine sample; Dr. Crina Balog for her support with data analysis; Rob Bruggink for providing essential input for producing the capillary desalter; Chris Pohl, Yan Liu, Victor Barretto, and Franck van Veen from Thermo Fisher Scientific for essential support of this research.

Open Access This article is distributed under the terms of the Creative Commons Attribution License which permits any use, distribution and reproduction in any medium, provided the original author(s) and source are credited.

\section{References}

1. Cantz M, Ulrich-Bott B (1990) Disorders of glycoprotein degradation. J Inherit Metab Dis 13:523-537
2. Lundblad A, Lundsten J, Nordén NE, Sjöblad S, Svensson S, Öckerman PA, Gehlhoff M (1978) Urinary abnormalities in fucosidosis. Eur J Biochem 83:513-521

3. Ockerman PA (1969) Diseases of glycoprotein storage. Lancet $1: 734$

4. Ockerman PA, Autio S, Norden NE (1973) Diagnosis of mannosidosis. Lancet 1:207-208

5. Winchester B (2005) Lysosomal metabolism of glycoproteins. Glycobiology 15:1R-15R

6. Cantz M, Gehler J, Spranger J (1977) Mucolipidosis I: increased sialic acid content and deficiency of an alpha- $N$-acetylneuraminidase in cultured fibroblasts. Biochem Biophys Res Commun 74:732-738

7. van Pelt J, Kamerling JP, Bakker HD, Vliegenthart JF (1991) A comparative study of sialyloligosaccharides isolated from sialidosis and galactosialidosis urine. J Inherit Metab Dis 14:730-740

8. Landing BH, Silverman FN, Chadwick DL, Lahey ME, Jacoby MD, Raig JM (1964) Familial neurovisceral lipidosis. An analysis of eight cases of a syndrome previously reported as "Hurler-variant," "Pseudo-Hurler," and "Tay-Sachs disease with visceral involvement". Am J Dis Child 108:503-522

9. Takahashi Y, Orii T (1989) Severity of GM1 gangliosidosis and urinary oligosaccharide excretion. Clin Chim Acta 179:153-162

10. Rubin M, Karpati G, Wolfe LS, Carpenter S, Klavins MH, Mahuran DJ (1988) Adult onset motor neuronopathy in the juvenile type of hexosaminidase A and B deficiency. J Neurol Sci 87:103119

11. Hoffman LM, Amsterdam D, Brooks SE, Schneck L (1977) Glycosphingolipids in fetal Tay-Sachs disease brain and lung cultures. J Neurochem 29:551-559

12. Mahuran DJ (1999) Biochemical consequences of mutations causing the GM2 gangliosidoses. Biochim Biophys Acta 1455:105138

13. Hou Y, Tse R, Mahuran DJ (1996) Direct determination of the substrate specificity of the alpha-active site in heterodimeric betahexosaminidase A. Biochemistry (Mosc) 35:3963-3969

14. Kolter T, Sandhoff K (2006) Sphingolipid metabolism diseases. Biochim Biophys Acta 1758:2057-2079

15. Strecker G, Herlant-Peers MC, Fournet B, Montreul J (1977) Structure of seven oligosaccharides excreted in the urine of a patient with Sandhoff's disease (GM2 gangliosidosis-variant O). Eur J Biochem 81:165-171

16. Warner TG, De Kremer RD, Applegarth D, Mock AK (1986) Diagnosis and characterization of GM 2 gangliosidosis type II (Sandhoff disease) by analysis of the accumulating $\mathrm{N}$-acetyl-glucosaminyl oligosaccharides with high performance liquid chromatography. Clin Chim Acta 154:151-164

17. Sewell AC (1979) An improved thin-layer chromatographic method for urinary oligosaccharide screening. Clin Chim Acta 92:411-414

18. Kin NM (1987) Comparison of the urinary glycoconjugates excreted by patients with type I and type II fucosidosis. Clin Chem 33:44-47

19. Schindler D, Kanzaki T, Desnick RJ (1990) A method for the rapid detection of urinary glycopeptides in alpha- $N$-acetylgalactosaminidase deficiency and other lysosomal storage diseases. Clin Chim Acta 190:81-91

20. An Y, Young SP, Hillman SL, Van Hove JL, Chen YT, Millington DS (2000) Liquid chromatographic assay for a glucose tetrasaccharide, a putative biomarker for the diagnosis of Pompe disease. Anal Biochem 287:136-143

21. Neville DC, Dwek RA, Butters TD (2009) Development of a single column method for the separation of lipid- and proteinderived oligosaccharides. J Proteome Res 8:681-687

22. Warner TG, Turner MW, Toone JR, Applegarth D (1986) Prenatal diagnosis of infantile GM 2 gangliosidosis type II (Sandhoff disease) by detection of $N$-acetylglucosaminyl-oligosaccharides in 
amniotic fluid with high-performance liquid chromatography. Prenat Diagn 6:393-400

23. Peelen GO, de Jong JG, Wevers RA (1994) HPLC analysis of oligosaccharides in urine from oligosaccharidosis patients. Clin Chem 40:914-921

24. Wuhrer M, Koeleman CA, Deelder AM, Hokke CH (2004) Normal-phase nanoscale liquid chromatography-mass spectrometry of underivatized oligosaccharides at low-femtomole sensitivity. Anal Chem 76:833-838

25. Bruggink C, Wuhrer M, Koeleman CA, Barreto V, Liu Y, Pohl C, Ingendoh A, Hokke CH, Deelder AM (2005) Oligosaccharide analysis by capillary-scale high-pH anion-exchange chromatography with on-line ion-trap mass spectrometry. J Chromatogr B Anal Technol Biomed Life Sci 829:136-143

26. Bruggink C, Poorthuis BJ, Piraud M, Froissart R, Deelder AM, Wuhrer M (2010) Glycan profiling of urine, amniotic fluid and ascitic fluid from galactosialidosis patients reveals novel oligosaccharides with reducing end hexose and aldohexonic acid residues. FEBS J 277:2970-2986

27. Packer NH, Lawson MA, Jardine DR, Redmond JW (1998) A general approach to desalting oligosaccharides released from glycoproteins. Glycoconj J 15:737-747

28. Rocklin RD, Clarke AP, Weitzhandler M (1998) Improved long-term reproducibility for pulsed amperometric detection of carbohydrates via a new quadruple-potential waveform. Anal Chem 70:1496-1501

29. Yamashita K, Kochibe N, Ohkura T, Ueda I, Kobata A (1985) Fractionation of L-fucose-containing oligosaccharides on immobilized Aleuria aurantia lectin. J Biol Chem 260:4688-4693

30. Michalski JC, Klein A (1999) Glycoprotein lysosomal storage disorders: alpha- and beta-mannosidosis, fucosidosis and alpha- $N$ acetylgalactosaminidase deficiency. Biochim Biophys Acta 1455:6984

31. Strecker G, Fournet B, Bouquelet S, Montreuil J, Dhondt JL, Farriaux JP (1976) Chemistry of urinary mannosides excreted in mannosidosis. Biochimie 58:579-586

32. Yamashita K, Tachibana Y, Mihara K, Okada S, Yabuuchi H, Kobata A (1980) Urinary oligosaccharides of mannosidosis. J Biol Chem 255:5126-5133

33. Matsuura F, Nunez HA, Grabowski GA, Sweeley CC (1981) Structural studies of urinary oligosaccharides from patients with mannosidosis. Arch Biochem Biophys 207:337-352

34. Yamashita K, Ohkura T, Okada S, Yabuuchi H, Kobata A (1981) Urinary oligosaccharides of GM1-gangliosidosis. Different excretion patterns of oligosaccharides in the urine of type 1 and type 2 subgroups. J Biol Chem 256:4789-4798

35. Klein A, Lebreton A, Lemoine J, Perini JM, Roussel P, Michalski JC (1998) Identification of urinary oligosaccharides by matrixassisted laser desorption ionization time-of-flight mass spectrometry. Clin Chem 44:2422-2428

36. Warner TG, Robertson AD, O'Brien JS (1983) Diagnosis of GM1 gangliosidosis based on detection of urinary oligosaccharides with high performance liquid chromatography. Clin Chim Acta 127:313326

37. Lundblad A (1978) In: Ginsburg V (ed) Methods in enzymology, vol 50. New York, Academic Press, p 226-235

38. Michalski JC (1996) In: Montreuil J, Vliegenthart JF, Schachter H (eds) Glycoproteins and disease. Amsterdam, Elsevier Science

39. Lowden JA, O'Brien JS (1979) Sialidosis: a review of human neuraminidase deficiency. Am J Hum Genet 31:1-18

40. van Pelt J, Kamerling JP, Vliegenthart JF, Hoogeveen AT, Galjaard H (1988) A comparative study of the accumulated sialic acidcontaining oligosaccharides from cultured human galactosialidosis and sialidosis fibroblasts. Clin Chim Acta 174:325-335

41. Spina E, Sturiale L, Romeo D, Impallomeni G, Garozzo D, Waidelich D, Glueckmann M (2004) New fragmentation mechanisms in matrix-assisted laser desorption/ionization time-of-flight/ time-of-flight tandem mass spectrometry of carbohydrates. Rapid Commun Mass Spectrom 18:392-398

42. Harvey DJ (2000) Electrospray mass spectrometry and fragmentation of $N$-linked carbohydrates derivatized at the reducing terminus. J Am Soc Mass Spectrom 11:900-915

43. Weiskopf AS, Vouros P, Harvey DJ (1998) Electrospray ionization-ion trap mass spectrometry for structural analysis of complex $N$-linked glycoprotein oligosaccharides. Anal Chem 70:4441-4447

44. Ramsay SL, Meikle PJ, Hopwood JJ, Clements PR (2005) Profiling oligosaccharidurias by electrospray tandem mass spectrometry: quantifying reducing oligosaccharides. Anal Biochem 345:30-46

45. Jardine I, Matsuura F, Sweeley CC (1984) Electron ionization mass spectra of reduced and permethylated urinary oligosaccharides from patients with mannosidosis. Biomed Mass Spectrom 11:562-568

46. Kunz C, Rudloff S, Baier W, Klein N, Strobel S (2000) Oligosaccharides in human milk: structural, functional, and metabolic aspects. Annu Rev Nutr 20:699-722

47. Finke B, Stahl B, Pfenninger A, Karas M, Daniel H, Sawatzki G (1999) Analysis of high-molecular-weight oligosaccharides from human milk by liquid chromatography and MALDI-MS. Anal Chem 71:3755-3762

48. Pfenninger A, Karas M, Finke B, Stahl B (2002) Structural analysis of underivatized neutral human milk oligosaccharides in the negative ion mode by nano-electrospray MS(n) (part 1: methodology). J Am Soc Mass Spectrom 13:1331-1340

49. Naarding MA, Ludwig IS, Groot F, Berkhout B, Geijtenbeek TB, Pollakis G, Paxton WA (2005) Lewis X component in human milk binds DC-SIGN and inhibits HIV-1 transfer to CD4+ T lymphocytes. J Clin Invest 115:3256-3264

50. Parkkinen J, Finne J (1983) Isolation and structural characterization of five major sialyloligosaccharides and a sialylglycopeptide from normal human urine. Eur J Biochem 136:355-361

51. Koseki M, Tsurumi K (1977) A convenient method for the isolation of 3'-sialyllactose from normal human urine. J Biochem 82:17851788

52. Hardy MR, Townsend RR (1988) Separation of positional isomers of oligosaccharides and glycopeptides by high-performance anionexchange chromatography with pulsed amperometric detection. Proc Natl Acad Sci U S A 85:3289-3293

53. Townsend RR, Hardy MR, Hindsgaul O, Lee YC (1988) Highperformance anion-exchange chromatography of oligosaccharides using pellicular resins and pulsed amperometric detection. Anal Biochem 174:459-470

54. Townsend RR, Hardy MR, Lee YC (1989) Separation of oligosaccharides using high-performance anion-exchange chromatography with pulsed amperometric detection. Methods Enzymol 179:6576

55. Vellodi A (2005) Lysosomal storage disorders. Br J Haematol 128:413431

56. Strecker G, Fournet B, Montreuil J (1978) Structure of the three major fucosyl-glycoasparagines accumulating in the urine of a patient with fucosidosis. Biochimie 60:725-734

57. Tsay GC, Dawson G (1976) Oligosaccharide storage in brains from patients with fucosidosis, GM1-gangliosidosis and GM2gangliosidosis (Sandhoff's disease). J Neurochem 27:733-740

58. Tsay GC, Dawson G, Sung SS (1976) Structure of the accumulating oligosaccharide in fucosidosis. J Biol Chem 251:5852-5859

59. Nishigaki M, Yamashita K, Matsuda J, Arashima S, Kobata A (1978) Urinary oligosaccharides of fucosidosis. Evidence of the occurrence of X-antigenic determinant in serum-type sugar chains of glycoproteins. J Biochem 84:823-834

60. Warner TG, deKremer RD, Mock AK, Sjoberg ER (1985) Characterization and analysis of branched-chain $N$-acetylglucosaminyl oligosaccharides accumulating in Sandhoff disease tissue. 
Evidence that biantennary bisected oligosaccharide side chains of glycoproteins are abundant substrates for lysosomes. J Biol Chem 260:6194-6199

61. Dorland L, Haverkamp J, Vliegenthart JF, Strecker G, Michalski JC, Fournet B, Spik G, Montreuil J (1978) 360-MHz 1 H nuclearmahnetic-resonance spectroscopy of sialyl-oligosaccharides from patients with sialidosis (mucolipidosis I and II). Eur J Biochem $87: 323-329$

62. Charlwood J, Tolson D, Camilleri P (1999) A detailed analysis of neutral and acidic carbohydrates in human milk. Anal Biochem 273:261-277

63. Fong BY, Ma K, McJarrow P (2011) Quantification of bovine milk oligosaccharides using liquid chromatography selected reaction monitoring-mass spectrometry. J Agric Food Chem 59: $9788-95$
64. Anumula KR (2006) Advances in fluorescence derivatization methods for high-performance liquid chromatographic analysis of glycoprotein carbohydrates. Anal Biochem 350:1-23

65. Ruhaak LR, Zauner G, Huhn C, Bruggink C, Deelder AM, Wuhrer M (2010) Glycan labeling strategies and their use in identification and quantification. Anal Bioanal Chem 397:3457-3481

66. Bruggink C, Maurer R, Herrmann H, Cavalli S, Hoefler F (2005) Analysis of carbohydrates by anion-exchange chromatography and mass spectrometry. J Chromatogr A 1085:104-109

67. Rabin S, Stillian JR, Barreto V, Friedman K, Toofan M (1993) New membrane-based electrolytic suppressor device for suppressed conductivity detection in ion chromatography. J Chromatogr 640:97-109

68. Liu Y, Srinivasan K, Pohl C, Avdalovic N (2004) Recent developments in electrolytic devices for ion chromatography. J Biochem Biophys Methods 60:205-232 